Louisiana State University

LSU Digital Commons

3-21-2017

\title{
Amphiphilic Polypeptoids Serve as the Connective Glue to Transform Liposomes into Multilamellar Structures with Closely Spaced Bilayers
}

\author{
Yueheng Zhang \\ Tulane University \\ Sunting Xuan \\ Louisiana State University \\ Olasehinde Owoseni \\ Tulane University \\ Marzhana Omarova \\ Tulane University \\ Xin Li \\ Louisiana State University
}

See next page for additional authors

Follow this and additional works at: https://digitalcommons.Isu.edu/chemistry_pubs

\section{Recommended Citation}

Zhang, Y., Xuan, S., Owoseni, O., Omarova, M., Li, X., Saito, M., He, J., McPherson, G., Raghavan, S., Zhang, D., \& John, V. (2017). Amphiphilic Polypeptoids Serve as the Connective Glue to Transform Liposomes into Multilamellar Structures with Closely Spaced Bilayers. Langmuir, 33 (11), 2780-2789. https://doi.org/ 10.1021/acs.langmuir.6b04190

This Article is brought to you for free and open access by the Department of Chemistry at LSU Digital Commons. It has been accepted for inclusion in Faculty Publications by an authorized administrator of LSU Digital Commons. For more information, please contact ir@lsu.edu. 


\section{Authors}

Yueheng Zhang, Sunting Xuan, Olasehinde Owoseni, Marzhana Omarova, Xin Li, Michelle E. Saito, Jibao He, Gary L. McPherson, Srinivasa R. Raghavan, Donghui Zhang, and Vijay T. John 
1 Amphiphilic Polypeptoids Serve as the Connective Glue to Transform Liposomes into

2 Multilamellar Structures with Closely Spaced Bilayers

3 Yueheng Zhang ${ }^{1}$, Sunting Xuan ${ }^{2}$, Olasehinde Owoseni ${ }^{1}$, Marzhana Omarova ${ }^{1}$, Xin $\mathrm{Li}^{3}$, Michelle

4 E. Saito ${ }^{1}$, Jibao $\mathrm{He}^{4}$, Gary L. McPherson ${ }^{5}$, Srinivasa R. Raghavan ${ }^{6}$, Donghui Zhang ${ }^{2 *}$ and Vijay 5 T. John ${ }^{1 *}$

6 1. Department of Chemical and Biomolecular Engineering

$7 \quad$ Tulane University, New Orleans, LA 70118, United States

$8 \quad$ Email: vj@ tulane.edu. Phone: (504) 865-5883. Fax: (504) 865-6744

9 2. Department of Chemistry and Macromolecular Studies Group

10 Louisiana State University, Baton Rouge, LA 70803, United States

$11 \quad$ Email: dhzhang @1su.edu. Phone: (225) 578-4893.

12 3. Louisiana Consortium for Neutron Scattering

13 Louisiana State University, Baton Rouge, LA 70803, United States

14 4. Coordinated Instrumentation Facility

15 Tulane University, New Orleans, LA 70118, United States

16 5. Department of Chemistry

17 Tulane University, New Orleans, LA 70118, United States

18 6. Department of Chemical and Biomolecular Engineering

19 University of Maryland, College Park, MD 20742, United States

20 *Corresponding authors. Vijay T. John, phone: 504-865-5883 e-mail: vj@tulane.edu. Donghui

21 Zhang, phone: 225-578-4893 email: dhzhang@1su.edu 
We report the ability of hydrophobically modified polypeptoids (HMPs), which are

3 amphiphilic pseudo-peptidic macromolecules, to connect across lipid bilayers and thus form

4 layered structures on liposomes. The HMPs are obtained by attaching hydrophobic decyl groups

5 at random points along the polypeptoid backbone. While native polypeptoids (with no

6 hydrophobes) have no effect on liposomal structure, the HMPs remodel the unilamellar

7 liposomes into structures with comparable diameters but with multiple concentric bilayers. The

8 transition from single bilayer to multiple bilayer structures is revealed by small angle neutron

9 scattering (SANS) and cryo-transmission electron microscopy (cryo-TEM). The spacing between

10 bilayers is found to be relatively uniform at $\sim 6.7 \mathrm{~nm}$. We suggest that the amphiphilic nature of

11 the HMPs explains the formation of multi-bilayered liposomes; i.e., the HMPs insert their

12 hydrophobic tails into adjacent bilayers and thereby serve as the connective glue between

13 bilayers. At higher HMP concentrations, the liposomes are entirely disrupted into much smaller

14 micelle-like structures through extensive hydrophobe insertion. Interestingly, these small

15 structures can reattach to fresh unilamellar liposomes and self-assemble to form new two-

16 bilayered liposomes. The two-bilayer liposomes in our study are reminiscent of two-bilayered

17 organelles such as the nucleus in eukaryotic cells. The observations have significance to 18 designing new nanoscale drug delivery carriers with multiple drugs on separate lipid bilayers and 19 extending liposome circulation times with entirely biocompatible materials. 
Liposomes are lipid based vesicles that are of significant interest in drug delivery

3 technologies due to their ability to encapsulate drug species and to be taken up by cells through

4 fusion with cell membranes. ${ }^{1-5}$ Ever since their discovery in 1965 by Bangham who noticed the

5 structural similarity to cell membranes, liposomes have been used in biophysical studies of

6 phospholipid bilayers and as cell membrane mimics. ${ }^{6-7}$ Newer applications of liposomes include

7 their use as coatings on biomedical devices, ${ }^{8}$ in single molecule spectroscopy and biosensing, ${ }^{9-10}$

8 and as submicron encapsulation systems in microfluidics. ${ }^{11}$

9 In many applications of liposomes, understanding the interaction of these vesicular systems

10 with polymers becomes extremely important, whether to enhance circulation lifetimes, to control

11 drug delivery rates, or to encapsulate liposomes in polymer matrices. This paper describes the

12 interaction of liposomes with a class of synthetic molecules known as polypeptoids that are

13 pseudo-peptidic materials with substituents attached to the nitrogen atoms of the polyglycine

14 backbone. ${ }^{12-13}$ These polymers have been of recent interest in chemical biology due to their

15 highly designable structure and their structural similarity to polypeptides. ${ }^{14-15}$ Unlike

16 polypeptides however, polypeptoids lack extensive hydrogen bonding and backbone chirality

17 due to the N-substitution, leading to a more flexible conformation and an enhanced resistance to

18 proteolysis. $^{16-18}$ Due to backbone degradability, biocompatibility, and processability,

19 polypeptoids are of promise in applications related to drug delivery carriers, tissue engineering

20 materials, and smart coatings. ${ }^{19-21}$ However, there is little information in the literature on

21 polypeptoid interaction with lipids and cell membranes.

22 The objective of this study is to define and control the interactions of a model liposome 23 system with two classes of polypeptoids in order to develop new concepts in liposome-polymer 
1 systems. The polymers chosen are (1) a polypeptoid containing the methoxyethyl (MeOEt)

2 groups exclusively as side chains, hereafter denoted as an unmodified polypeptoid (UMP) and (2)

3 a polypeptoid where about $26 \%$ of the MeOEt groups are randomly substituted by the n-decyl

4 (C10) groups hereafter denoted as a hydrophobically modified polypeptoid (HMP) as shown in

5 Figure 1a. Both polypeptoids can be synthesized through the synthetic route shown in Figure1b.

a<smiles>COCCN(CC(=O)NCc1ccccc1)C(C)(C)O</smiles>

Unmodified polypeptoid (UMP)

$\mathrm{Mw}=12509 \mathrm{~g} / \mathrm{mol}$

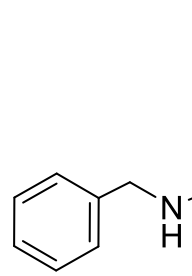

Hydrophobically modified polypeptoid (HMP) $\mathrm{Mw}=13939 \mathrm{~g} / \mathrm{mol}$<smiles>CCCCC/C=C\C/C=C\CCCCCCCC(=O)OCC(C)(F)OC(=O)CCCCCCC/C=C\CCCCC</smiles>

L- $\alpha$-phosphatidylcholine (PC)
6

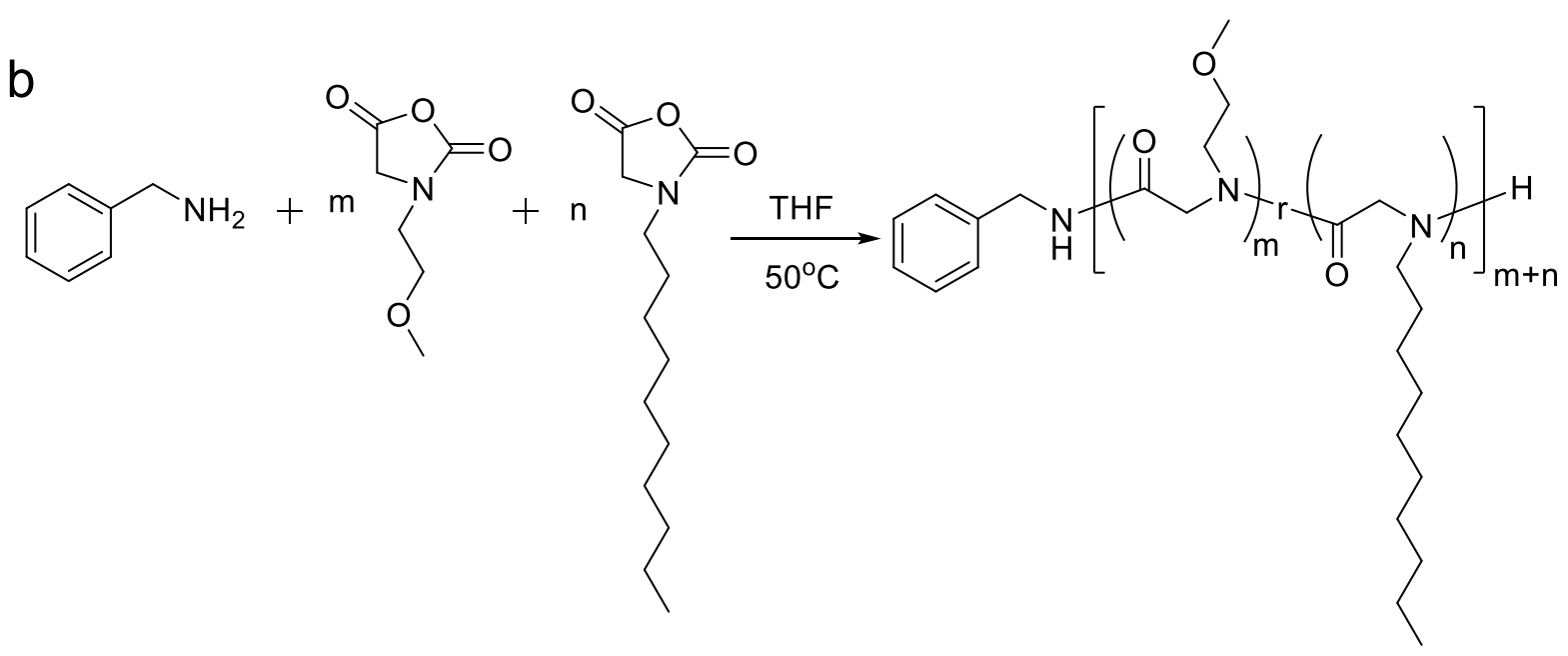

Figure 1. (a) Chemical structures of unmodified polypeptoid (UMP), hydrophobically modified polypeptoid (HMP) and L- $\alpha$-phosphatidylcholine (PC). (b) Synthetic route to the polypeptoids. 
The presence of the hydrophobic alkyl groups leads to the attachment of polymers to the

2 lipid bilayers through the insertion of alkyl groups into the bilayer as a consequence of

3 hydrophobic interaction. Such insertions of hydrophobic groups into the bilayer are inherent

4 properties of polymeric surfactants (polysoaps) ${ }^{22-23}$, peptide amphiphiles ${ }^{24}$ and lipopolypeptoids

525 . In the case of a lipid conjugated polyethylene glycol (PEG) (such as 1,2-distearoyl-sn-

6 glycero-3-phosphoethanolamine-N-amino (polyethylene glycol), DSPE-PEG), the polymer chain

7 is attached to the liposome surface through insertion of the hydrophobic distearoyl group into the

8 lipid bilayer. ${ }^{26}$ This is important for extending the circulation times of liposomes for drug

9 delivery. Bis alkyl hydrophobes attached to PEG have been shown by Auguste and coworkers to

10 bind more strongly to the bilayer in comparison to monoalkyl hydrophobes. ${ }^{27}$ Work by Shen and

11 coworkers have shown the potential for bis alkyl lipopolymers to tether lipid bilayers to

12 surfaces. $^{28}$ When multiple alkyl groups are present on long-chain polymers, the liposomes are

13 connected into a network by the polymer chains. ${ }^{29}$ Hydrophobic interactions are intrinsic in

14 understanding the effects of surfactants on lipid bilayers, where liposomes and cell membranes

15 have been found to break down through fluidization of the bilayer and eventual dissolution of the

16 lipids into mixed lipid-surfactant micelles. ${ }^{30-33}$

17

19 liposomes which we elaborate on, in this paper. Specifically, HMPs seem to stitch together

20 vesicle bilayers, leading to multilayered liposomes with small and relatively uniform spacings

21 between their concentric bilayers. Such structures have not been observed before. The formation

22 of these multilayered structures is confirmed by a combination of SANS and cryo-TEM. 


\section{Materials.}

L- $\alpha$-phosphatidylcholine (PC) was purchased from Avanti Polar Lipids. Deuterium oxide was purchased from Cambridge Isotope Laboratories. Deionized (DI) water generated by ELGA reverse osmosis water purification system (MEDICA 15BP) with a resistance of $18.2 \mathrm{M} \Omega \cdot \mathrm{cm}$ was used in all experiments. All chemicals were used as received unless otherwise noted. $\mathrm{N}$ decyl $N$-carboxyanhydride ( $N$-decyl NCA) in $53-57 \%$ yield and $N$-methoxyethyl $N$ carboxyanhydride ( $\mathrm{N}$-MeOEt NCA) in $43-49 \%$ yield were synthesized by adapting previously reported procedures, as shown in Supporting Information Scheme S1 (route I and II). ${ }^{34-35}$

\section{Synthesis of Polypeptoids.}

The polypeptoids were synthesized in a glove box through benzyl amine-initiated ringopening polymerization of the corresponding $N$-substituted $N$-carboxylanhydride (R-NCA) monomers. The unmodified polypeptoid (UMP), poly( $N$-methoxyethyl glycine) (PNMeOEtG), was synthesized by polymerization of $N$-methoxyethyl NCA, whereas the hydrophobically modified polypeptoid (HMP), poly[( $N$-methoxyethyl glycine)-r- $(N$-decyl glycine $)]$ (P(NMeOEtG-r-NDG)), was synthesized by copolymerization of $N$-methoxyethyl NCA and $N$ decyl NCA (Figure 1b). Both UMP and HMP are designed to reach polymerization degree of 100. In a typical synthesis of HMP, stock solutions of $N$-methoxyethyl NCA (1.3 ml, $0.4 \mathrm{M}$, $0.52 \mathrm{mmol})$ and $N$-decyl NCA $(0.3 \mathrm{ml}, 0.4 \mathrm{M}, 0.13 \mathrm{mmol})$ in THF were mixed in a small vial. Benzyl- $\mathrm{NH}_{2} / \mathrm{THF}$ stock solution $(70 \mu \mathrm{l}, 92.7 \mathrm{mM}, 6.5 \mu \mathrm{mol})$ was added to the above mixture and heated at $50{ }^{\circ} \mathrm{C}$ for $48 \mathrm{~h}$. Aliquots were taken and analyzed by ${ }^{1} \mathrm{H}$ NMR spectroscopy to check conversion. The polymer was precipitated out by adding excess hexanes, collected by 
1 filtration, and dried under vacuum to obtain the final product as a white solid $(61.6 \mathrm{mg}, 88.2 \%$

2 yield).

3 NMR and Size Exclusion Chromatography (SEC) Analysis of the Polypeptoids.

$4 \quad{ }^{1} \mathrm{H}$ and ${ }^{13} \mathrm{C}\left\{{ }^{1} \mathrm{H}\right\}$ NMR spectra were obtained using a Bruker AV-400 Nanobay spectrometer

5 (400 MHz for ${ }^{1} \mathrm{H}$ NMR and $100 \mathrm{MHz}$ for ${ }^{13} \mathrm{C}\left\{{ }^{1} \mathrm{H}\right\}$ NMR) at $298 \mathrm{~K}$. Chemical shifts $(\delta)$ given in

6 parts per million (ppm) were referenced to proton impurities or the ${ }^{13} \mathrm{C}$ isotopes of deuterated

7 solvents $\left(\mathrm{CDCl}_{3}, \mathrm{CD}_{2} \mathrm{Cl}_{2}\right.$, and $\left.\mathrm{D}_{2} \mathrm{O}\right) .{ }^{1} \mathrm{H}$ NMR (Supporting Information Figure S1) and ${ }^{13} \mathrm{C}\left\{{ }^{1} \mathrm{H}\right\}$

8 NMR (Supporting Information Figure S2) of the monomers are included in the Supporting

9 Information, respectively. SEC analysis of the polypeptoids were performed using an Agilent 1200 system (Agilent 1200 series degasser, isocratic pump, autosampler and column heater)

11 equipped with three Phenomenex $5 \mu \mathrm{m}, 300 \times 7.8 \mathrm{~mm}$ columns [100 $\AA, 1000 \AA$ and Linear (2)],

12 a Wyatt OptilabrEX differential refractive index (DRI) detector with a $690 \mathrm{~nm}$ light source, and a Wyatt DAWN EOS multiangle light scattering (MALS) detector (GaAs 30 $\mathrm{mW}$ laser at $\lambda=690$ $14 \mathrm{~nm}$ ). DMF with $0.1 \mathrm{M} \mathrm{LiBr}$ was used as the eluent at a flow rate of $0.5 \mathrm{~mL} \cdot \mathrm{min}^{-1}$. The column and detector temperature was set at $25^{\circ} \mathrm{C}$. All data analysis was performed using Wyatt Astra $\mathrm{V}$ 5.3 software. The polydispersity index (PDI) were obtained by conventional SEC analysis with a

17 calibration curve. The calibration curve was constructed from twenty three pauci-disperse 18 polystyrene standards $\left(\mathrm{M}_{\mathrm{n}}=590 \mathrm{~g} \cdot \mathrm{mol}^{-1}-1472 \mathrm{~kg} \cdot \mathrm{mol}^{-1}\right.$, Polymer Laboratories, Inc. $)$ using Astra's column calibration template. Relative PDI was then calculated using Astra's conventional calibration template. The sample for SEC analysis was prepared as below: $130 \mu 1$

21 reaction mixture of $\mathrm{HMP}$ (or UMP) was added to $1 \mathrm{ml}$ of DMF with $0.1 \mathrm{M} \mathrm{LiBr}$ and mixed

22 thoroughly. This mixture was directly injected into SEC instrument for analysis. 


\section{Liposome Preparation.}

2 L- $\alpha$-phosphatidylcholine (PC) liposomes were prepared using thin film hydration method. ${ }^{36}$

$3 \quad 0.1 \mathrm{~g}$ PC was first dissolved in a round bottom flask by $15 \mathrm{ml}$ mixed solution of chloroform and

4 methanol at a volume ratio of 2:1. The solvent was then evaporated using a rotary evaporator

5 (Buchi R-205) at room temperature under 100 mbar for 3 hours to form a thin lipid film. The

6 pressure was further reduced to $6 \mathrm{mbar}$ for $30 \mathrm{~min}$ to remove solvent residual. The obtained thin

7 lipid film was hydrated with DI water at $50{ }^{\circ} \mathrm{C}$. The aqueous suspension was transferred to a 8 syringe and extruded 21 times through a $100 \mathrm{~nm}$ polycarbonate membrane to acquire liposomes

9 with an average diameter of $100 \mathrm{~nm}$.

\section{Preparation of Liposome and Polypeptoid Mixtures.}

DI water was used to dilute the liposome stock suspension to $0.5 \%$. HMP solutions at concentrations of $0.25 \%, 0.5 \%, 0.75 \%$ and $1 \%$ were mixed with a $0.5 \%$ liposome suspension at 1:1 volume ratio, respectively, to acquire solutions with the liposome concentration fixed at $0.25 \%$ and varying concentrations of HMP. The resulting mixtures were labeled as LIP0125HMP, LIP025HMP, LIP0375HMP and LIP05HMP denoting the final overall concentrations of HMP. Liposomes were also incubated with UMP at overall concentrations of $0.25 \%$ and $0.5 \%$ as

17 negative controls, denoted as LIP025UMP and LIP05UMP, respectively. The liposome solution 18 with the overall concentration of $0.25 \%$ was used as the blank control, denoted as LIP. 19 Essentially all samples had the same concentration of lipid (0.25\%) and the studies were done 20 with varying concentrations of polymers. All samples were incubated for at least 12 hours prior 21 to instrumental characterization. All concentrations are reported as wt $\%$. 
SANS Data Collection and Reduction.

The SANS experiments were carried out on the extended-Q range small angle neutron scattering (EQ-SANS) diffractometer at the Spallation Neutron Source (SNS) at the Oak Ridge

5 National Laboratory (ORNL). All samples were prepared with pure deuterium oxide to generate

6 sufficient scattering contrast. The samples were loaded into $2 \mathrm{~mm}$ path-length quartz banjo cells

7 (Hellma, Germany) and placed in a 42-position sample chamber. Measurements were taken at 8 room temperature $\left(20{ }^{\circ} \mathrm{C}\right)$. The instrument was operated in a $60 \mathrm{~Hz}$ mode with a neutron 9 wavelength $(\lambda)$ range of $2.5 \AA-6.1 \AA$. The sample-to-detector distance was set to $4 \mathrm{~m}$. This 10 configuration provides an effective q-range of $0.009 \AA^{-1}-0.44 \AA^{-1}$. The scattering vector, $q$, is 11 defined by $q=4 \pi[\sin (\theta / 2)] / \lambda$ where $\theta$ is the scattering angle.

The reduction of SANS data was performed using MantidPlot software following standard 13 procedures. ${ }^{37}$ The data were corrected for instrument dark current, detector sensitivity, incident 14 beam normalization, sample transmission and solvent background. A calibrated standard 15 provided by ORNL, Porasil B, was used to obtain the scale factor which was used to convert the 16 data into absolute intensity units $\left(\mathrm{cm}^{-1}\right)$. Reduced data were azimuthally averaged using 17 MantidPlot to generate scattering intensity per unit volume, $I(q)$, as a function of $q$.

\section{Cryo-TEM Imaging.}

Cryo-TEM imaging was done on an FEI G2 F30 Tecnai TEM operated at $150 \mathrm{kV}$. To 20 prepare the sample, a 300-mesh lacey carbon grid (Electron Microscopy Sciences) was picked up 21 by a tweezer and mounted on the plunging station of an FEI Vitrobot. $10 \mu$ l of the solution was 22 applied to the grid. The excess liquid was blotted by filter paper attached to arms of the Vitrobot 
1 for 2 seconds to form a thin film. The sample was then vitrified by plunging into liquid ethane.

2 The vitrified sample was finally transferred onto a single tilt cryo specimen holder for imaging.

3 RESULTS AND DISCUSSION

4 Polypeptoid Characterization through ${ }^{1} \mathrm{H}$ NMR and Size Exclusion Chromatography

5 (SEC).

6 The compositions of UMP and HMP were determined by ${ }^{1} \mathrm{H}$ NMR spectroscopy

7 (Supporting Information Figure S3). The number average degree of polymerization (DP) of

8 UMP was determined by the integration of methylene group in the backbone at 4.52, 4.41, 4.18,

$94.10 \mathrm{ppm}$ relative to the integration of benzyl end-group at 7.24 and $7.31 \mathrm{ppm}$. The DP of HMP

10 was determined by the integration of methyl group in the side chain and the integration of

11 methylene groups in the backbone relative to the integration of benzyl end-group at 7.24 and

$127.31 \mathrm{ppm}$. The integrations give UMP a DP of 106 and HMP a DP of 100 containing $26 \mathrm{~mol} \%$

$13 N$-decyl glycine units, corresponding to molecular weight of $12509 \mathrm{~g} / \mathrm{mol}$ for UMP and 13939

$14 \mathrm{~g} / \mathrm{mol}$ for HMP. The size exclusion chromatographic (SEC) analysis indicated the monomodal

15 and narrow distribution of polymer molecular weight for both UMP and HMP (Supporting

16 Information Figure S4), with low PDI of 1.09 and 1.08, respectively.

17 Small Angle Neutron Scattering (SANS).

As the first step to the characterization of the interactions between the polymers and

19 liposomes, we carried out SANS experiments on the individual systems with the results shown in

20 Figure 2. As shown here, the data are separated by a scale factor of 0.1 for clarity and ease in

21 visualization especially at the larger q values. The scattering intensity, $I(q)$, is determined by the 
1 structure factor, $S(q)$, and the form factor, $P(q)$. In dilute, noninteracting system, $S(q)$ is close to

2 1. Therefore, $I(q)$ is determined by $P(q) .{ }^{38}$

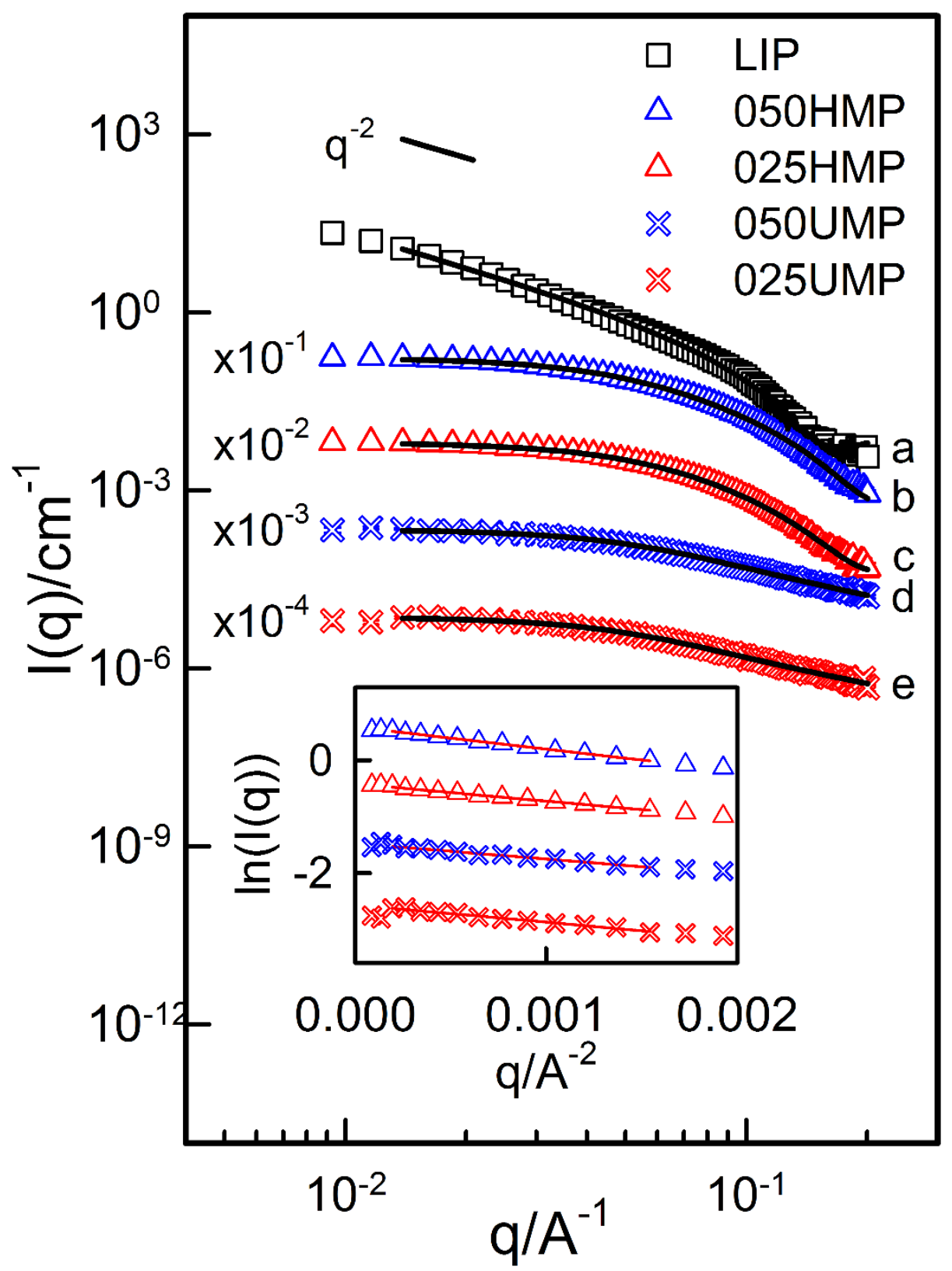

5

Figure 2. Scattering profiles of $0.25 \%$ liposome (LIP, a), $0.5 \%$ HMP (050HMP, b), $0.25 \%$ HMP (025HMP, c), 0.5\% UMP (050UMP, d) and 0.25\% UMP (025UMP, e) in $\mathrm{D}_{2} \mathrm{O}$. The curves are 
1 scaled and separated by a factor of 0.1 . The solid lines are best-fit results to the corresponding

2 models. The inset shows the Guinier plots (ln(I) vs. $\left.\mathrm{q}^{2}\right)$ of the HMP and UMP samples.

3 Curve a in Figure 2 shows the scattering profile of $0.25 \%$ liposome suspension (LIP). For a

4 detailed analysis of the form factor, the scattering data was fitted to the PolyCoreMultiShell

5 model developed by the National Institute of Standards and Technology (NIST) using IgorPro

6 software. When $\mathrm{N}$ (model parameter, number of shells) is fixed at $1, \mathrm{P}(\mathrm{q})$ can be described as ${ }^{39-}$

$7 \quad 40$

$$
P(q)=\frac{\text { scale }}{V_{\text {shell }}}\left[\frac{3 V_{1}\left(\rho_{1}-\rho_{2}\right) J_{1}\left(q R_{1}\right)}{q R_{1}}+\frac{3 V_{2}\left(\rho_{2}-\rho_{\text {solv }}\right) J_{1}\left(q R_{2}\right)}{q R_{2}}\right]^{2}+b k g
$$

9 where scale is a scale factor, ${ }^{39} V_{\text {shell }}$ is the volume of the shell, $V_{l}$ is the volume of the core, $V_{2}$ is the total volume, $R_{l}$ is the radius of the core, $R_{2}$ is the outer radius of the shell, $\rho_{l}$ is the scattering

11 length density of the core, $\rho_{2}$ is the scattering length density of the shell, $\rho_{\text {solv }}$ is the scattering

12 length density of the solvent, bkg is the background level, and $J_{1}(x)=(\sin x-x \cos x) / x^{2}$.

13 With polydispersity in the core radius being considered, the form factor is averaged over the 14 Schultz distribution ${ }^{38}$

$$
P(q)=\int f(R) P(q, R) d R
$$

where $f$ is the distribution function, $R$ is the core radius, $R_{0}$ is the average core radius, $\Gamma(x)$ is the gamma function, and $z$ is the polydispersity index. The polydispersity, $p$, is related to $z$ through

$$
f(R)=\left(\frac{z+1}{R_{0}}\right)^{z+1} \frac{R^{z}}{\Gamma(z+1)} \exp \left[-(z+1) \frac{R}{R_{0}}\right]
$$

20 The scattering profile of the liposome system (Figure 2, curve a) indicates a $\mathrm{q}^{-2}$ dependence in 21 the decay at low q range, which is characteristic of non-interacting liposomes. ${ }^{32}$ The solid line 
1 shows the best-fit of the scattering data of liposome. The fitting results show a liposome diameter

2 of $99.4 \mathrm{~nm}$ with a polydispersity of 0.17 . The shell thickness, or the lipid bilayer thickness, is

$3 \quad 3.68 \mathrm{~nm}$, which is close to the reported value in the literature. ${ }^{41-42}$

$4 \quad$ We found that the scattering curves for HMP samples were best fitted to the Flexible 5 Cylinder model. ${ }^{43-44}$ For UMP, the curve was also fitted by the Gaussian Coil model, but we 6 retained the excellent fit of the Flexible Cylinder model to provide a direct comparison of 7 parameters with HMP. In both cases we assumed that the contour length $L>>$ the Kuhn length $b$.

8 The results are presented in Figure 2 as solid black lines. L was calculated based on 9 polymerization degree and was fixed in the Flexible Cylinder model calculations to estimate b 10 and the axial radius of the polymer aggregates, $R_{\text {cyl }}$. The radius of gyration, $R_{g}$, of each sample 11 was also calculated from the model fit results. ${ }^{43}$ The results are included in Table 1.

Table 1. Model Fitting Parameters for SANS Data

\begin{tabular}{ccccccc}
\hline Sample & \multicolumn{2}{c}{ Components } & $\mathbf{R}_{\mathbf{c y l}}^{\mathrm{a}}(\mathbf{n m})$ & $\mathbf{L}^{\mathrm{b}}(\mathbf{n m})$ & $\mathbf{b}^{\mathrm{c}}(\mathbf{n m})$ & $\mathbf{R}_{\mathbf{g}}(\mathbf{n m})$ \\
\hline 025HMP & - & $0.25 \%$ HMP & 1.7 & 38.0 & 0.76 & 3.0 \\
\hline 050HMP & - & $0.5 \%$ HMP & 1.7 & 38.0 & 1.08 & 3.4 \\
\hline 025UMP & - & $0.25 \%$ UMP & 0.6 & 39.4 & 1.00 & 3.0 \\
\hline 050UMP & - & $0.5 \%$ UMP & 0.6 & 39.4 & 0.87 & 2.9 \\
\hline LIP050HMP & $0.25 \%$ liposome & $0.5 \%$ HMP & 2.0 & 38.0 & 3.43 & 4.7
\end{tabular}
${ }^{a} \mathrm{R}_{\text {cyl }}$ : cylinder radius; ${ }^{b} \mathrm{~L}$ : contour length; ${ }^{c} \mathrm{~b}$ : Kuhn length; ${ }^{\mathrm{d}} \mathrm{R}_{\mathrm{g}}$ values calculated based on fitting results
of Flexible Cylinder model.

The inset to Figure 2 shows the empirical Guinier plots where data in the range of 0.00014 $\AA^{-2}<q^{2}<0.0016 \AA^{-2}$ were used in the calculation for $R_{g}$. The results of the Guinier analysis are listed in Table 1 and show very reasonable agreement with the $\mathrm{R}_{\mathrm{g}}$ values calculated from the 
1 model fitting results. To summarize Table 1 , the values of $R_{g}$ between UMP and HMP show

2 insignificant differences at both concentrations and we infer that the state of the polymers over

3 this range of concentration remains essentially the same. The fact that the form factor fits the low

$4 \quad$ q range in these dilute systems may additionally imply that there is minimal intermolecular

5 aggregation. As shown in subsequent discussion, significant changes in $\mathrm{R}_{\mathrm{g}}$ values are obtained

6 through the interaction of HMP with lipid bilayers.

7 To explore the interactions between liposomes and polypeptoids, we prepared solutions

8 containing $0.25 \%$ liposomes with $0.25 \%$ and $0.5 \%$ UMP as control samples. The SANS data for

9 these samples (LIP025UMP and LIP050UMP, respectively) are shown in Figure 3. At low q, the

10 slope of the scattering curve remains -2 when liposomes are mixed with UMPs, which is

11 indicative that the bilayer structure is maintained when liposome is incubated with UMPs. We

12 note that the scattering intensity of the mixtures is essentially just the concentration weighted

13 summation of the individual components with the scattering from the liposomes dominating the

14 pattern at low q. These observations indicate that there is no structural change in the system

15 induced by UMP and that UMP and liposomes exist as independent entities in solution. 


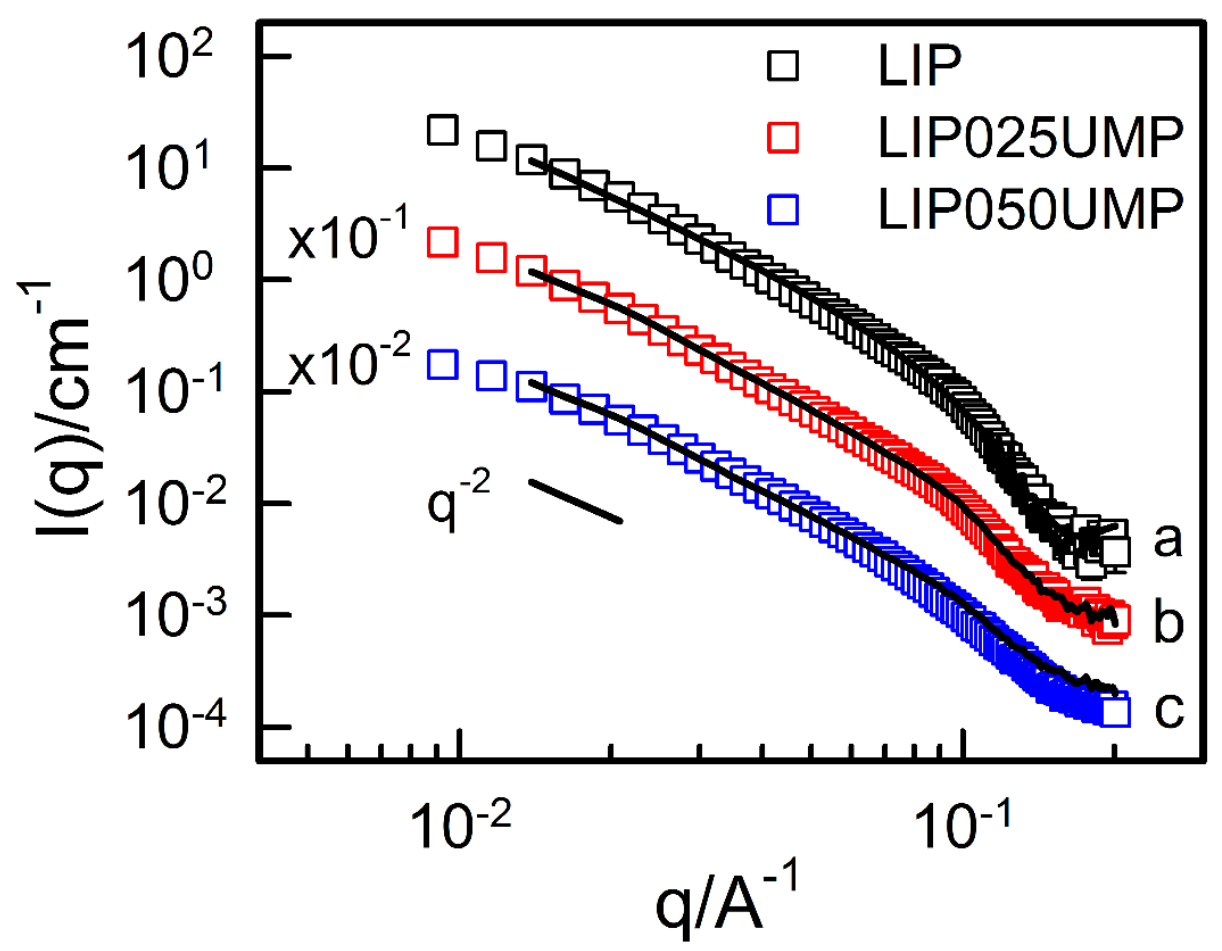

Figure 3. Scattering profiles of $0.25 \%$ liposomes incubated with $0 \%$ UMP (LIP, a), $0.25 \%$ UMP (LIP025UMP, b) and 0.5\% UMP (LIP050UMP, c). The scattering curves are separated by a scale factor of 0.1 for clarity. Addition of UMP has little effect on the scattering pattern which is essentially a linear summation of the scattering data of the individual components.

Figure 4 illustrates the scattering patterns of mixtures of liposomes and HMP, and it is immediately clear that there are significant changes in the patterns in comparison to the scattering from LIP. With the addition of HMP, the slope of the scattering curve at the low $\mathrm{q}$ range flattens significantly, indicating changes in the liposome bilayer structure. Along with this, a significantly decreased intensity at low q range was observed for each sample with HMP added compared to the control sample (Supporting Information Figure S5 shows the unscaled data for the plots of Figure 4). We additionally see a Bragg diffraction peak around $q=0.092 \AA^{-1}$ at an HMP concentration of $0.125 \%$ which shifts to slightly higher q and decreases in intensity with increased addition of HMP. The drop in scattering intensity at low q with the addition of more scattering entities (HMPs) and the observation of the Bragg peak are counter intuitive and are not 
1 easily fit using models for neutron scattering profiles. The drop in scattering intensity points to a

2 decrease in the number density of the larger scattering entities (liposomes) and the Bragg peak

3 suggests the occurrence of layered structures with relatively uniform spacing. But to better

4 understand these SANS observations, we have used cryo-TEM, and the imaging results for all

5 samples used in SANS analysis are described below. 


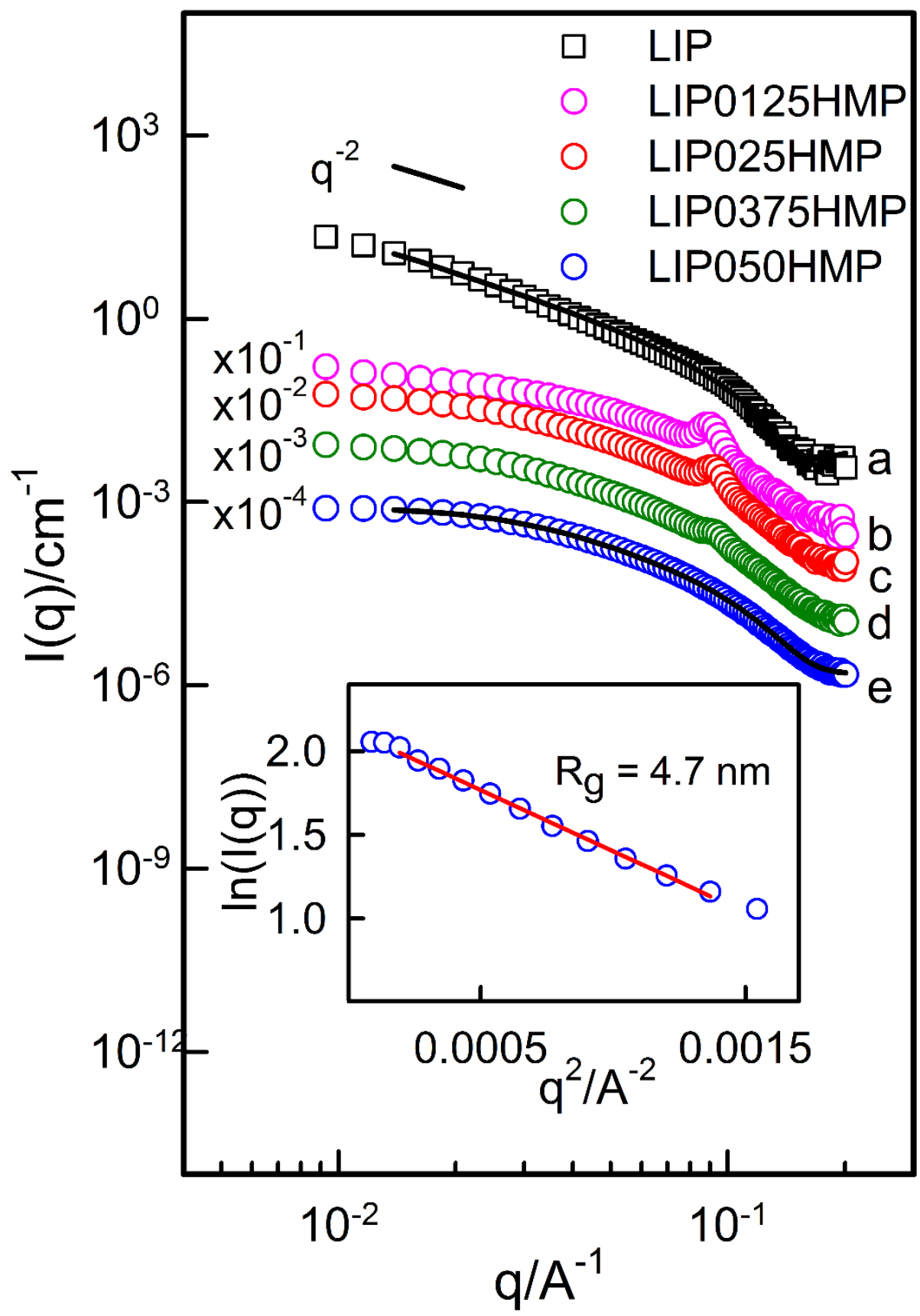

Figure 4. SANS data of $0.25 \%$ liposomes incubated with $0 \%$ HMP (LIP, a), $0.125 \%$ HMP (LIP0125HMP, b), 0.25\% HMP (LIP025HMP, c), 0.375\% HMP (LIP0375HMP, d) and $0.5 \%$ HMP (LIP050HMP, e). The scattering curves are separated by a scale factor of 0.1 for clarity. The black solid lines show best-fit results to the corresponding models. The inset shows the Guinier plot of the scattering data of LIP0125HMP giving an $\mathrm{R}_{\mathrm{g}}$ of $4.7 \mathrm{~nm}$. 


\section{Cryo Transmission Electron Microscopy of Microstructures}

Figure 5a and b show cryo-TEM images of the control samples containing liposomes alone (Figure 5a) and liposomes incubated with UMP (Figure 5b) where we have done higher resolution imaging to verify the unilamellarity of the liposomes. The clear observations are that the unilamellar PC liposomes are unchanged by the addition of UMP, in agreement with the SANS results. It is, therefore, evident that the liposomes are stable in the presence of UMPs and that liposome-UMP interactions do not affect liposome structure.
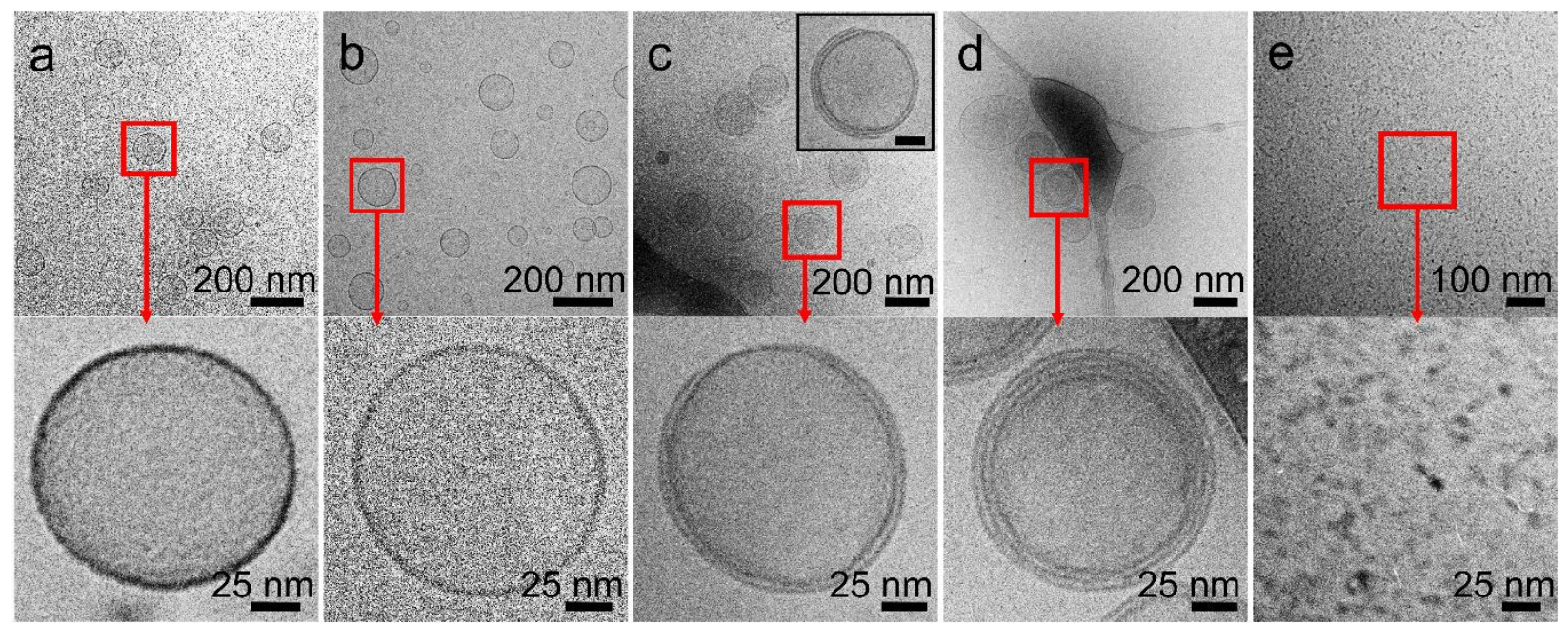

Figure 5. Cryo-TEM images of $0.25 \%$ liposome incubated in DI water (LIP, a), $0.25 \%$ UMP (LIP025UMP, b), 0.125\% HMP (0125HMP, c), 0.25\% HMP (LIP025HMP, d) and 0.5\% HMP (LIP050HMP, e), and corresponding high magnification images below them (red square). While addition of UMP has no effect on the liposome structure, addition of HMP to liposome suspension results in a transition from unilamellar structure to two-bilayer (c) and multiplebilayer (d) structures, and finally a solubilization of liposome (e). The inset in c indicates liposomes with multiple layer structures also exist in LIP0125HMP sample but with a small population. Scale bar of the inset is $50 \mathrm{~nm}$.

Figure $5 \mathrm{c}$ to $5 \mathrm{e}$ illustrates the cryo-TEM images of liposomes incubated with varying concentrations of HMP. With $0.125 \%$ HMP addition, while there is no significant change in the size of liposomes, there is the interesting visualization of a second lipid layer, with the higher resolution image of Figure 6c showing that the layer is incomplete. We also observe liposomes with multiple bilayers (inset to Figure 5c). With cryo-TEM it is not possible to determine the 
1 number density of imaged objects as number density varies at different locations on the grid.

2 Nevertheless, we observed a reduced density of liposomes over the TEM field of view. The

3 observation of a reduced number density becomes more pronounced with the addition of HMP to

4 a concentration of $0.25 \%$, and again we see the presence of liposomes with multiple bilayers

5 (Figures 5d). In all these cases, the number of bilayers varies, and the formed additional layers

6 are not necessarily complete and uniform. The average distance between each two adjacent

7 layers (center to center distance) in the high magnification image of Figure 5d was measured

8 using Nano Measure software. Multiple measurements were taken at different locations, and the

9 results were averaged. The measured average spacing distance is $6.8 \pm 0.9 \mathrm{~nm}$, which agrees

10 very well with the observed Bragg peak at a q value of $0.092 \AA$ corresponding to a d spacing of

$116.8 \mathrm{~nm}$. To our knowledge, this is the first observation of liposomes with two or more bilayers

12 with a relatively uniform spacing of a few $\mathrm{nm}$.

13 When the HMP concentration is further increased to $0.5 \%$, the SANS data shows the loss of

14 the Bragg peak (curve e in Figure 4). On the cryo-TEM, we are unable to see any liposomes in

15 the TEM field of view indicating a complete disassembly of liposomes. However, we see clear

16 evidence of small nanoscale structures (Figure 5e). A close examination of the images reveals

17 that some of the shapes in the 2-D image appear somewhat circular with dimensions around $5 \mathrm{~nm}$,

18 and some appear elongated as small wormlike structures with a $5 \mathrm{~nm}$ thickness and a length of

19 10-15 nm. The circular dots typically have a higher contrast perhaps indicative of a coiled

20 globular structure or of rods vitrified with axes parallel to the beam. The scattering curve at this

21 HMP concentration can be again modeled through the Flexible Cylinder model (Table 1). The $\mathrm{R}_{\mathrm{g}}$

22 value calculated from the model fitting result is $5.1 \mathrm{~nm}$, while the Guinier plot (inset to Figure 4)

23 shows the $\mathrm{R}_{\mathrm{g}}$ value of $4.7 \mathrm{~nm}$ and we note that these values are much smaller than liposomal 
1 dimensions but appreciably larger than the values for UMP and HMP alone. These suggest that

2 the liposomes are converted into such new nanoscale structures upon addition of HMPs. We also

3 note that HMP when added to the hydrating solution at $0.5 \mathrm{wt} \%$ prevents the formation of the

4 large liposomes typically observed prior to extrusion and leads to the formation of small

5 fragments similar to Figure 5e, thus indicating the generality of the phenomenon of liposome 6 disruption.

7 Both SANS and cryo-TEM reveal the important observation that HMP disrupts liposomes 8 and at sufficiently high concentrations completely disassembles these vesicular structures. But 9 most importantly, it is an additional conclusion that at intermediate concentrations, the HMPs 10 induce the liposomes to fuse which leads to the creation of two or more bilayer structures on 11 surviving liposomes. We propose that it is the formation of such multilayer liposomes that gives 12 rise to the Bragg peak in the scattering.

13 While the above results relate to equilibrated samples, cryo-TEM makes it possible to gain 14 an understanding of the dynamics of the structural transformations by contacting the liposomes 15 with HMP and rapidly vitrifying the systems at varying incubation times. Thus, $0.25 \%$ liposome 16 was incubated with $0.25 \%$ HMP (LIP0250HMP) for $1 \mathrm{~min}, 20 \mathrm{~min}$, and $45 \mathrm{~min}$ after which they 17 were vitrified for cryo-TEM imaging. The sample incubated for 1 min (Figure 6a) shows 18 significant deformation of liposomes and open bilayer structures. After 20 min (Figure 6b), we 19 see evidence of the formation of two bilayer structures and liposome rearrangement. At this stage, 20 while the liposomes are multilayered, the spacing between bilayers is not uniform and 21 considerably larger than the equilibrium value. At $45 \mathrm{~min}$, liposomes with multi-layered structure 22 are observed (Figure 6c) with the bilayer spacing down to $\sim 7 \mathrm{~nm}$. This result suggests that the 23 formation of multilayer liposomes undergoes a stepwise process. After HMP incorporates in the 
bilayers, liposomes first partially break up and form open bilayer structures or large sheets

2 (incomplete liposomes). The incomplete liposomes attach to surviving liposomes through hydrophobe insertion into bilayers to form multilayer structures. HMP thus serves as a

4 connective material between the liposome bilayer and additional bilayers. This is distinctly different from small molecule surfactants (with single hydrophobic tail) that simply fluidize and eventually break up liposomes into mixed micelles of surfactant and lipid. [24, 27]
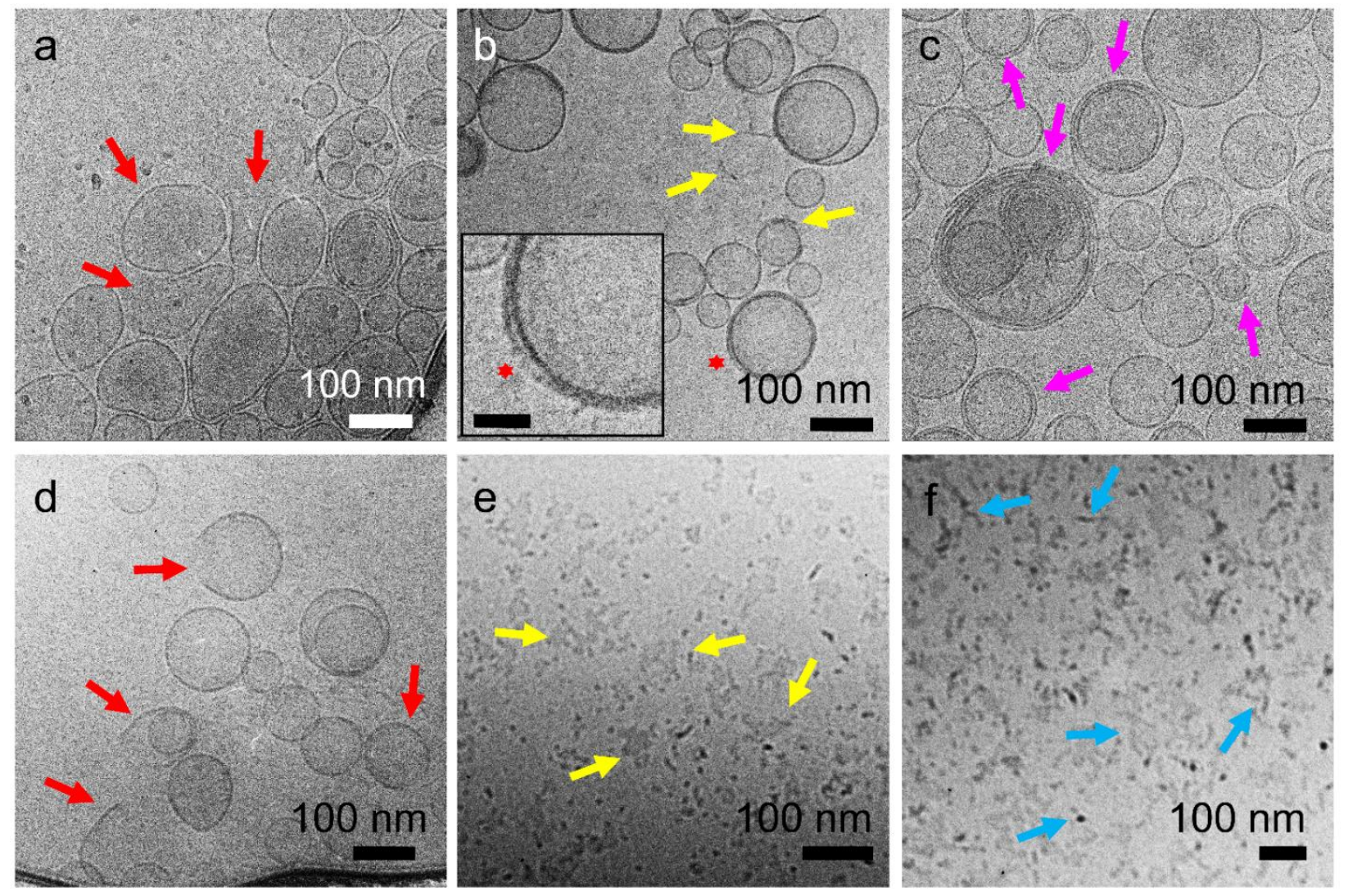

Figure 6. (a-c) Cryo-TEM images of $0.25 \%$ liposomes incubated with $0.25 \%$ HMP (LIP025HMP) for $1 \mathrm{~min}$ (a), $20 \mathrm{~min}$ (b) and $45 \mathrm{~min}$ (c). In a, arrows show deformed liposomes and open bilayer structures; in b, arrows show fragments of liposomes and the inset shows the two bilayers of the starred liposome (scale bar is $50 \mathrm{~nm}$ ); and in c, arrows show liposomes with multibilayered structures. (d-f) Cryo-TEM images of $0.25 \%$ liposomes incubated with $0.5 \%$ HMP (LIP050HMP) for $1 \mathrm{~min}$ (d), $20 \mathrm{~min}$ (e) and $45 \mathrm{~min}$ (f). Arrows in d show open bilayer structures; arrows in e show small fragments and the sample has no remnant intact liposomes; and in $\mathrm{f}$, small nanoscale structures are observed (typical structures are pointed to by the arrows).

Figure 6d-f illustrate the identical experiments but with $0.5 \%$ HMP (LIP050HMP). Within 
1 min of incubation, we see the coexistence of small fragments and debris (Figure 6e). With 45

2 min of incubation (Figure 6f), a complete breakdown of the liposomes occurs with the same

3 suspended nanoscale structures observed as in Figure 5e.

4 Based on the SANS and cryo-TEM observations we propose the following mechanism for

5 the results of liposome-HMP interactions as shown in Figure 7. At the lower HMP

6 concentrations, a fraction of the liposomes is broken into large fragments by the insertion of

7 hydrophobes that have a sufficient local concentration in the bilayer to fluidize and disrupt the

8 bilayer. Such softening of lipid bilayers through hydrophobe insertion is considered the dominant

9 mechanism of liposome disruption by surfactants where the traditional model involves

10 dissolution of liposomes into mixed lipid-surfactant micelles. ${ }^{[35-38]}$ Polymeric hydrophobes-

11 containing amphiphiles such as HMPs are clearly distinct as they can bridge between lipid

12 bilayers. Thus at the lower HMP concentrations, fragments with attached HMPs can reattach to

13 remnant intact liposomes either through insertion of some of the free hydrophobes into the

14 bilayer of intact liposomes or through interaction with other HMP chains that may be attached to

15 the unbroken liposome (Figure 7b). We propose that the attachment of these fragments may

16 continue to the formation of multiple layers and the spacings are sufficiently periodic to give rise

17 to the constructive interference in neutron diffraction leading to the observed peak at a d spacing

18 of $6.8 \mathrm{~nm}$. At high HMP concentrations, incorporation of additional HMPs into lipid bilayers

19 results in a solubilization of lipid bilayers by forming lipid-HMP small elongated structures that 20 are modeled by flexible cylinders. 

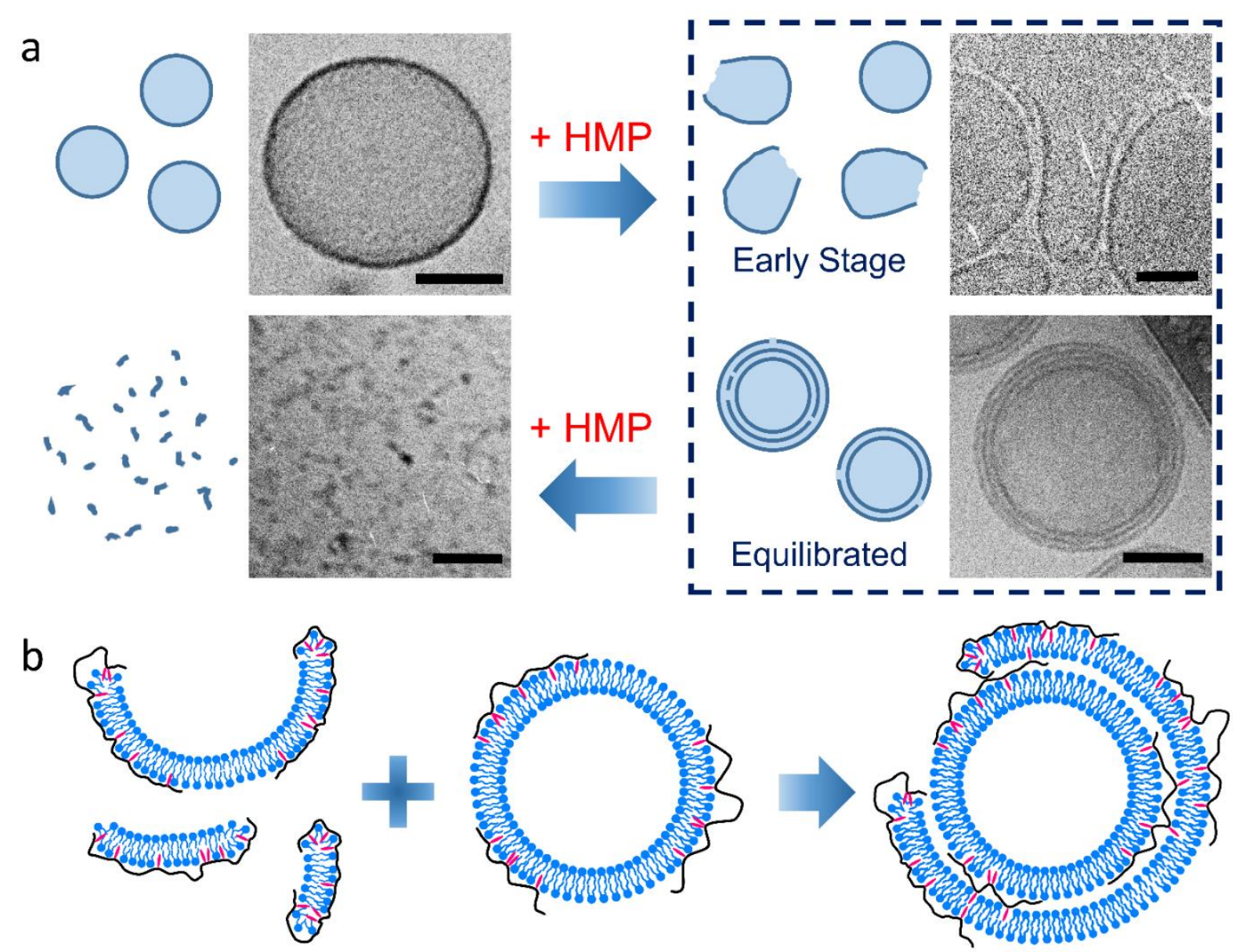

Figure 7. (a) Schematic of the formation of multiple-bilayer liposomes and the solubilization of liposomes induced by HMP (scale bars in all the images are $50 \mathrm{~nm}$ ). (b) Illustration of HMP bridging the fragments and lipid bilayer of a liposome by hydrophobe insertion forming twobilayer structure.

The hypothesis that fragments with the hydrophobes containing polymer can be hooked on to lipid bilayers was tested through the reverse experiment of contacting the completely broken down liposome system as shown in Figure 5e with a suspension of fresh intact liposomes The experiment was carried out by mixing the LIP050HMP solution with a fresh batch of liposomes (LIP) at a volume ratio of 1:2 (Figure 8a). Remarkably, incomplete layers form on the surface of the new liposome, and it is difficult to visualize any remaining broken up structures (Figure 8b). The two-bilayer structure is therefore reconstructed. The continuity of the second layer implies 
a
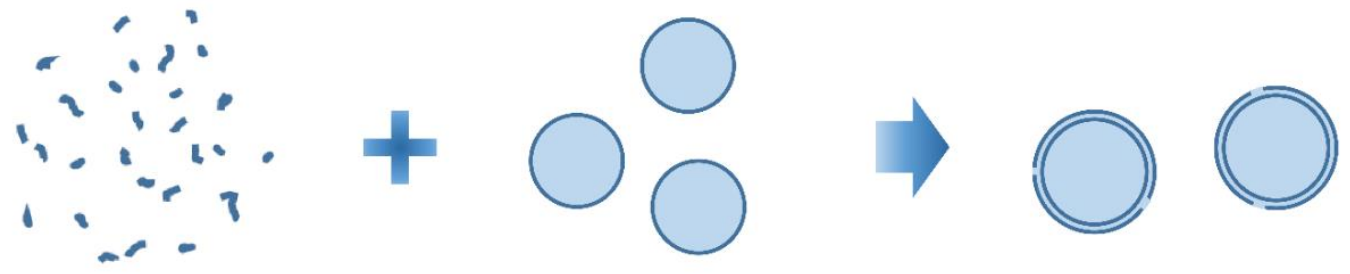

$\mathrm{b}$
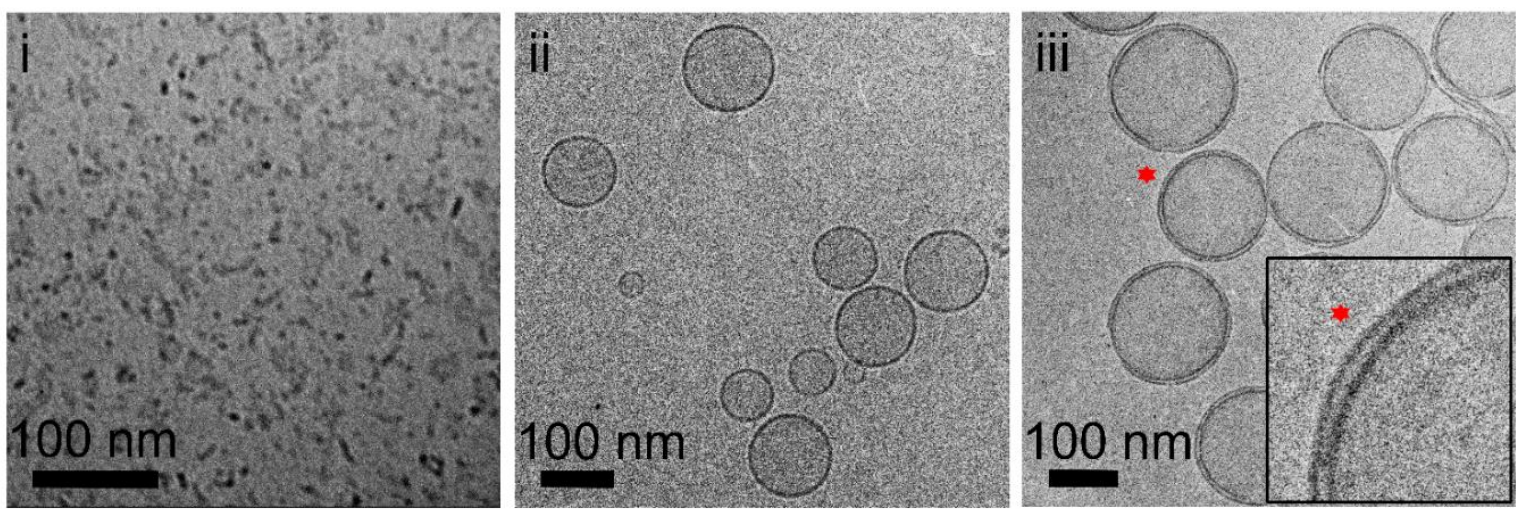

C
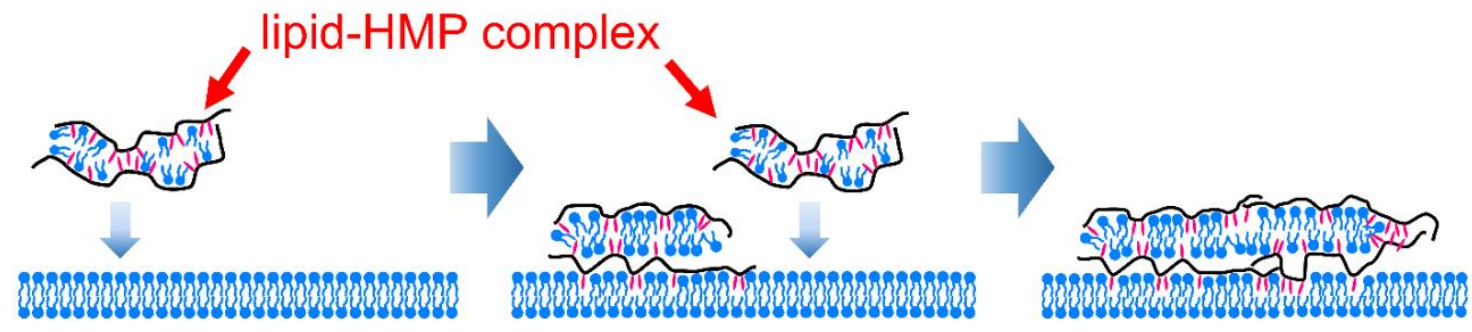

Figure 8. (a) Schematic of the experiment showing addition of a lipid-HMP complex solution (LIP050HMP) to a fresh liposome suspension (LIP) at a volume ratio of 1:2. (b) Cryo-TEM images show lipid-HMP complex (i), fresh liposome (ii) and reconstructed bilayers on bare liposomes (iii). The inset in iii shows the reconstructed bilayer on liposome (identified by the asterisk with scale bar $25 \mathrm{~nm}$ ). (c) Schematic of building up bilayer structure from lipid-HMP complexes. When the lipid-HMP complexes contact bare liposomes, HMP incorporates into the bilayers by hydrophobe insertion into the fresh bilayers. The assembly of such attachment results in formation of additional lipid bilayers. 
2 In this study, we have demonstrated that the introduction of decyl groups to a hydrophilic

3 polypeptoid backbone can induce interactions between the modified polypeptoid and liposomes

4 through the insertion of hydrophobes into the lipid bilayer. This leads to a breakdown of the

5 liposome structural integrity and a gradual solubilization of the liposome into mixed aggregates

6 of lipids and the modified polypeptoid. In this respect, the phenomenon is similar to the

7 interaction of molecular surfactants with liposomes. A distinct difference however, is the ability

8 of the HMP polymeric amphiphile to attach fragments onto existing and surviving liposomes to

9 create additional layers on the surface of liposomes. The polymer chain serves as the connecting

10 material between lipid containing fragments and liposomal bilayers allowing a buildup of

11 bilayers on the surface of a liposome. In contrast to small molecule surfactants, such polymeric

12 surfactants have membrane interaction properties that may be adjusted both through polymer

13 concentration and the relative degree of hydrophobe substitution on the chain backbone.

The potential to build bilayers onto liposomes using a designed connective polymeric amphiphile has significant technical implications. These concepts may have relevance in the attachment of drug-containing lipid entities to cell membranes and to vesicle systems. The ability

17 of HMPs to remodel liposomes is a novel aspect of multilayer self-assembly with significant applications to using liposomes as multiple drug carriers. For example, it may be possible to load 19 the interior of the liposome with water soluble drugs, and the bilayer with a hydrophobic drug.

20 Subsequently the patchwork addition of a second bilayer containing additional drug or diagnostic

21 components may lead to multilamellar liposomes with multiple therapeutics or sensing agents.

22 We also note that the double bilayer structure has a resemblance to those found in organelles 
1 such as in the nuclear membrane of eukaryotic cells, although the bilayer spacing here is smaller.

2 Our continuing research seeks to understand these effects in realizing new applications.

3

4 Supporting Information. NMR spectrum of HMP and UMP, SEC chromatograms of HMP and 5 UMP, unscaled SANS data of liposomes incubated with varying concentrations of HMPs at low 6 q range.

7

8 ACKNOWLEDGEMENTS

$9 \quad$ We acknowledge Tianyi Yu (LSU) for assistance in materials characterization through NMR 10 and melting point determination. This work was supported by the U.S. Department of Energy 11 under EPSCoR Grant No. DE-SC0012432. The Research at Oak Ridge National Laboratory's

12 Spallation Neutron Source was sponsored by the Scientific User Facilities Division, Office of 13 Basic Energy Sciences, U.S. Department of Energy. We are grateful to Dr. William Heller 14 (ORNL) for assistance in the small angle neutron scattering studies. 


\section{REFERENCES}

(1) Peyman, G. A.; Ganiban, G. J. Delivery Systems for Intraocular Routes. Adv. Drug Delivery Rev. 1995, 16, 107-123.

(2) Dhule, S. S.; Penfornis, P.; He, J. B.; Harris, M. R.; Terry, T.; John, V.; Pochampally, R. The Combined Effect of Encapsulating Curcumin and C6 Ceramide in Liposomal Nanoparticles against Osteosarcoma. Mol. Pharmaceutics 2014, 11, 417-427.

(3) Dhule, S. S.; Penfornis, P.; Frazier, T.; Walker, R.; Feldman, J.; Tan, G.; He, J. B.; Alb, A.; John, V.; Pochampally, R. Curcumin-Loaded Gamma-Cyclodextrin Liposomal Nanoparticles as Delivery Vehicles for Osteosarcoma. Nanomedicine 2012, 8, 440-451.

(4) Khuller, G. K.; Kapur, M.; Sharma, S. Liposome Technology for Drug Delivery against Mycobacterial Infections. Curr. Pharm. Des. 2004, 10, 3263-3274.

(5) Allen, T. M.; Austin, G. A.; Chonn, A.; Lin, L.; Lee, K. C. Uptake of Liposomes by Cultured Mouse Bone-marrow Macrophages - Influence of Liposome Composition and Size. Biochim. Biophys. Acta 1991, 1061, 56-64.

(6) Ricchelli, F.; Jori, G.; Gobbo, S.; Tronchin, M. Liposomes as Models to Study the Distribution of Porphyrins in Cell Membranes Biochim. Biophys. Acta 1991, 1065, 42-48.

(7) Yoshina-Ishii, C.; Chan, Y. H. M.; Johnson, J. M.; Kung, L. A.; Lenz, P.; Boxer, S. G. Diffusive Dynamics of Vesicles Tethered to a Fluid Supported Bilayer by Single Particle Tracking. Langmuir 2006, 22, 5682-5689.

(8) Lunelli, L.; Pasquardini, L.; Pederzolli, C.; Vanzetti, L.; Anderle, M. Covalently Anchored Lipid Structures on Amine-Enriched Polystyrene. Langmuir 2005, 21, 8338-8343. (9) Cooper, M. A.; Hansson, A.; Lofas, S.; Williams, D. H. A Vesicle Capture Sensor Chip for Kinetic Analysis of Interactions with Membrane-Bound Receptors. Anal. Biochem. 2000, 277, 196-205. 
(10) Boukobza, E.; Sonnenfeld, A.; Haran, G. Immobilization in Surface-Tethered Lipid

Vesicles as a New Tool for Single Biomolecule Spectroscopy. J. Phys. Chem. B 2001, 105,

$3 \quad 12165-12170$.

4 (11) Christensen, S. M.; Stamou, D. Surface-Based Lipid Vesicle Reactor Systems:

$5 \quad$ Fabrication and Applications. Soft Matter 2007, 3, 828-836.

6 (12) Lahasky, S. H.; Hu, X.; Zhang, D. Thermoresponsive Poly(alpha-peptoid)s: Tuning the

7 Cloud Point Temperatures by Composition and Architecture. ACS Macro Lett. 2012, 1, 580-584.

8 (13) Gangloff, N.; Ulbricht, J.; Lorson, T.; Schlaad, H.; Luxenhofer, R. Peptoids and

9 Polypeptoids at the Frontier of Supra- and Macromolecular Engineering. Chem. Rev. 2016, 116, $10 \quad 1753-1802$.

11 (14) Sun, J.; Zuckermann, R. N. Peptoid Polymers: A Highly Designable Bioinspired 12 Material. ACS Nano 2013, 7, 4715-4732.

13 (15) Zhang, D.; Lahasky, S. H.; Guo, L.; Lee, C.-U.; Lavan, M. Polypeptoid Materials:

14 Current Status and Future Perspectives. Macromolecules 2012, 45, 5833-5841.

15 (16) Murnen, H. K.; Rosales, A. M.; Dobrynin, A. V.; Zuckermann, R. N.; Segalman, R. A. 16 Persistence Length of Polyelectrolytes with Precisely Located Charges. Soft Matter 2013, 9, 901798.

18 (17) Rosales, A. M.; Murnen, H. K.; Kline, S. R.; Zuckermann, R. N.; Segalman, R. A. 19 Determination of the Persistence Length of Helical and Non-helical Polypeptoids in Solution. 20 Soft Matter 2012, 8, 3673-3680.

21 (18) Miller, S. M.; Simon, R. J.; Ng, S.; Zuckermann, R. N.; Kerr, J. M.; Moos, W. H.

22 Proteolytic Studies of Homologous Peptide and N-substituted Glycine Peptoid Oligomers.

23 Bioorg. Med. Chem. Lett. 1994, 4, 2657-2662. 
(19) Xuan, S.; Lee, C.-U.; Chen, C.; Doyle, A. B.; Zhang, Y.; Guo, L.; John, V. T.; Hayes, D.; Zhang, D. Thermoreversible and Injectable ABC Polypeptoid Hydrogels: Controlling the Hydrogel Properties through Molecular Design. Chem. Mater. 2016, 28, 727-737.

(20) Lu, L.; Lahasky, S. H.; Zhang, D.; Garno, J. C. Directed Growth of Polymer Nanorods Using Surface-Initiated Ring-Opening Polymerization of N-Allyl N-Carboxyanhydride. ACS Appl. Mater. Interfaces 2016, 8, 4014-4022.

(21) Hörtz, C.; Birke, A.; Kaps, L.; Decker, S.; Wächtersbach, E.; Fischer, K.; Schuppan, D.; Barz, M.; Schmidt, M. Cylindrical Brush Polymers with Polysarcosine Side Chains: A Novel Biocompatible Carrier for Biomedical Applications. Macromolecules 2015, 48, 2074-2086. (22) Laschewsky, A. Molecular concepts, self-organisation and properties of polysoaps. In Polysoaps/Stabilizers/Nitrogen-15 NMR; Springer Berlin Heidelberg: Berlin, Heidelberg, 1995, pp 1-86.

(23) Raffa, P.; Wever, D. A. Z.; Picchioni, F.; Broekhuis, A. A. Polymeric Surfactants: Synthesis, Properties, and Links to Applications. Chem. Rev. 2015, 115, 8504-8563.

(24) Trent, A.; Marullo, R.; Lin, B.; Black, M.; Tirrell, M. Structural properties of soluble peptide amphiphile micelles. Soft Matter 2011, 7, 9572-9582.

(25) Douy, A.; Gallot, B. New amphipathic lipopeptides, 1. Synthesis and mesomorphic structures of lipopeptides with polysarcosine peptidic chains. Die Makromolekulare Chemie 1986, $187,465-483$.

(26) Immordino, M. L.; Dosio, F.; Cattel, L. Stealth liposomes: review of the basic science, rationale, and clinical applications, existing and potential. Int. J. Nanomed. 2006, 1, 297-315. 
1 (27) Auguste, D. T.; Prud'homme, R. K.; Ahl, P. L.; Meers, P.; Kohn, J. Association of

2 hydrophobically-modified poly(ethylene glycol) with fusogenic liposomes. Biochim. Biophys.

3 Acta, Biomembr. 2003, 1616, 184-195.

4 (28) Shen, W. W.; Boxer, S. G.; Knoll, W.; Frank, C. W. Polymer-Supported Lipid Bilayers

5 on Benzophenone-Modified Substrates. Biomacromolecules 2001, 2, 70-79.

6 (29) Chen, Y.; Javvaji, V.; MacIntire, I. C.; Raghavan, S. R. Gelation of Vesicles and

7 Nanoparticles using Water-Soluble Hydrophobically Modified Chitosan. Langmuir 2013, 29,

$8 \quad 15302-15308$.

9 (30) Shoji, Y.; Igarashi, T.; Nomura, H.; Eitoku, T.; Katayama, K. Liposome Solubilization 10 Induced by Surfactant Molecules in a Microchip. Anal. Sci. 2012, 28, 339-343.

11 (31) Lichtenberg, D.; Ahyayauch, H.; Alonso, A.; Goñi, F. M. Detergent Solubilization of 12 Lipid Bilayers: a Balance of Driving Forces. Trends Biochem. Sci 2013, 38, 85-93.

13 (32) Lichtenberg, D.; Ahyayauch, H.; Goni, F. M. The Mechanism of Detergent Solubilization 14 of Lipid Bilayers. Biophys. J. 2013, 105, 289-299.

15 (33) Mattei, B.; França, A. D. C.; Riske, K. A. Solubilization of Binary Lipid Mixtures by the 16 Detergent Triton X-100: The Role of Cholesterol. Langmuir 2015, 31, 378-386.

17 (34) Lee, C.-U.; Smart, T. P.; Guo, L.; Epps, T. H.; Zhang, D. Synthesis and Characterization 18 of Amphiphilic Cyclic Diblock Copolypeptoids from N-Heterocyclic Carbene-Mediated 19 Zwitterionic Polymerization of N-Substituted N-carboxyanhydride. Macromolecules 2011, 44, $20 \quad 9574-9585$.

21 (35) Xuan, S.; Gupta, S.; Li, X.; Bleuel, M.; Schneider, G. J.; Zhang, D. Synthesis and 22 Characterization of Well-defined PEGylated Polypeptoids as Protein-resistant Polymers. 23 Biomacromolecules 2017. 
(36) Holder, G. E.; McGary, C. M.; Johnson, E. M.; Zheng, R.; John, V. T.; Sugimoto, C.;

2 Kuroda, M. J.; Kim, W.-K. Expression of the Mannose Receptor CD206 in HIV and SIV

3 Encephalitis: A Phenotypic Switch of Brain Perivascular Macrophages with Virus Infection. $J$.

$4 \quad$ Neuroimmune Pharmacol. 2014, 9, 716-726.

5 (37) Liu, Y.; Li, M.; Yang, Y.; Xia, Y.; Nieh, M.-P. The Effects of Temperature, Salinity,

6 Concentration and PEGylated Lipid on the Spontaneous Nanostructures of Bicellar Mixtures.

7 Biochim. Biophys. Acta, Biomembr. 2014, 1838, 1871-1880.

8 (38) Lee, J.-H.; Gustin, J. P.; Chen, T.; Payne, G. F.; Raghavan, S. R. Vesicle-Biopolymer

9 Gels: Networks of Surfactant Vesicles Connected by Associating Biopolymers. Langmuir 2005, $10 \quad 21,26-33$.

11 (39) Guinier, A.; Fournet, G. Small Angle Scattering of X-rays; John Wiley and Sons: New 12 York, 1955.

13 (40) Pabst, G.; Kučerka, N.; Nieh, M. P.; Katsaras, J. Liposomes, Lipid Bilayers and Model 14 Membranes: From Basic Research to Application; CRC Press: Boca Raton, 2014.

15 (41) Lewis, B. A.; Engelman, D. M. Lipid Bilayer Thickness Varies Linearly with Acyl Chain 16 Length in Fluid Phosphatidylcholine Vesicles. J. Mol. Biol. 1983, 166, 211-217.

17 (42) Balgavý, P.; Dubničková, M.; Kučerka, N.; Kiselev, M. A.; Yaradaikin, S. P.; Uhríková, 18 D. Bilayer Thickness and Lipid Interface Area in Unilamellar Extruded 1,2-

19 diacylphosphatidylcholine Liposomes: a Small-Angle Neutron Scattering Study. Biochim.

20 Biophys. Acta, Biomembr. 2001, 1512, 40-52.

21 (43) Pedersen, J. S.; Schurtenberger, P. Scattering Functions of Semiflexible Polymers with 22 and without Excluded Volume Effects. Macromolecules 1996, 29, 7602-7612. 
1 (44) Chevrier, M.; Houston, J. E.; Kesters, J.; Van den Brande, N.; Terry, A. E.; Richeter, S.;

2 Mehdi, A.; Coulembier, O.; Dubois, P.; Lazzaroni, R.; Van Mele, B.; Maes, W.; Evans, R. C.;

3 Clement, S. Self-Assembled Conjugated Polyelectrolyte-Surfactant Complexes as Efficient

4 Cathode Interlayer Materials for Bulk Heterojunction Organic Solar Cells. J. Mater. Chem. A

$5 \quad 2015,3,23905-23916$. 